Published in final edited form as:

J Cardiovasc Electrophysiol. 2014 July ; 25(7): 781-786. doi:10.1111/jce.12437.

\title{
Compatibility of Electroanatomical Mapping Systems with a Concurrent Percutaneous Axial Flow Ventricular Assist Device
}

Vaibhav R. Vaidya, MBBS ${ }^{1}$, Christopher V. DeSimone, MD, $\mathrm{PhD}^{2}$, Malini Madhavan, $\mathrm{MBBS}^{2}$, Amit Noheria, MBBS, SM$^{2}$, Mohammed Shahid, BS ${ }^{3}$, Jacob Walters, BS ${ }^{4}$, Dorothy J. Ladewig, BA ${ }^{5}$, Susan B. Mikell, BA ${ }^{2}$, Susan B. Johnson, BS ${ }^{2}$, Scott H. Suddendorf, $\mathbf{R T}^{6}$, and Samuel J. Asirvatham, MD ${ }^{2,7}$

1 Department of Internal Medicine, Mayo Clinic, Rochester, MN

${ }^{2}$ Division of Cardiovascular Diseases, Department of Internal Medicine, Mayo Clinic, Rochester, MN

${ }^{3}$ University of Pennsylvania, Department of Bioengineering

${ }^{4}$ St. Jude Medical, Minneapolis, MN

${ }^{5}$ Department of Research Administrative Services, Mayo Clinic, Rochester, MN

${ }^{6}$ Department of Surgical Research, Mayo Clinic, Rochester, MN

${ }^{7}$ Department of Pediatrics and Adolescent Medicine, Mayo Clinic, Rochester, MN

\section{Abstract}

Background-Hemodynamic instability hinders activation and entrainment mapping during ventricular tachycardia (VT) ablation. The Impella 2.5 microaxial flow device (MFD) (Abiomed Inc., Danvers, MA, USA) is used to prevent hemodynamic instability during electrophysiologic study. However, electromagnetic interference (EMI) generated by this device can preclude accurate electroanatomic mapping.

Methods-Impella was placed in the left ventricle of 7 canines for circulatory support. Electroanatomic mapping during sinus rhythm, ventricular pacing, and ventricular fibrillation (VF) was performed using magnet- (Carto3, Biosense Webster Inc., Diamond Bar, CA, USA) and impedance- (EnSite Velocity System/EnSite NavX, St. Jude Medical Inc., St. Paul, MN, USA) based systems. Distance from device to points with severe EMI precluding acquisition was compared to points with mild/no EMI. Two methods were used to reduce EMI: 1) titration of MFD performance, and 2) impedance-only mapping combined with manual annotation of activation.

Results-Severe EMI did not occur during impedance-based mapping. Severe EMI was observed using Carto3 at 9.4\% of all points attempted at maximum performance level (P8) of device. Severe EMI occurred at points closer to device $(40.1 \pm 16.8 \mathrm{~mm})$ vs. $(55.5 \pm 20.0 \mathrm{~mm})$ for

Corresponding Author: Samuel J. Asirvatham, M.D., Division of Cardiovascular Diseases, Mayo Clinic College of Medicine, 200 First Street SW, Rochester, MN 55905, Tel: 507-293-3376, Fax: 507-255-2550, asirvatham.samuel@ mayo.edu.

No disclosures. 
mild/no EMI, $\mathrm{p}<0.0001$. Severe EMI using Carto3 was resolved by either 1) reduction of performance from P8 to P6 or 2) impedance-only mapping with manual annotation

Conclusion-Concurrent use of MFD caused EMI to prevent acquisition of points with magnetbased mapping. Predictors for EMI were distance from device and performance level. Temporary reductions to P6 or impedance-only mapping are two methods to resolve EMI.

\section{Keywords}

ventricular tachycardia; catheter ablation; Impella; electroanatomical mapping systems; impedance; magnetic

\section{Introduction}

Catheter ablation of ventricular tachycardia (VT) is commonly performed for the management of symptomatic VT, frequent ICD shocks, and VT electrical storm. ${ }^{1-6}$ Detailed activation and entrainment mapping of VT is not always possible in hemodynamically unstable VT, and can lead to sub-optimal catheter ablation outcomes. ${ }^{4,5,7}$ Non-contact mapping, substrate-based mapping, pace-mapping, and antiarrhythmic therapy have been utilized in patients with unstable VT with varying degrees of success. ${ }^{8-13}$ Several case reports and series have described the use of various mechanical circulatory assist devices to maintain hemodynamic stability during VT ablation. ${ }^{14-21}$ Although the long term efficacy of this approach remains unknown, retrospective studies have reported comparable efficacy to substrate-based mapping. ${ }^{15}$

The Impella 2.5 microaxial flow device (MFD) (Abiomed Inc., Danvers, MA, USA) allows for activation mapping while maintaining adequate tissue perfusion during VT ablation. ${ }^{17,19-21}$ The Carto3 system (Biosense Webster Inc., Diamond Bar, CA, USA) is a magnet-based electroanatomic mapping system that uses low-level magnetic fields emitted by coils placed beneath the patient and a location sensor on the mapping catheter for electroanatomic mapping. The motion of the impeller within the microaxial flow device can result in electromagnetic interference (EMI) when a magnet-based system is used for electroanatomical mapping. EMI can impair accurate catheter localization, electroanatomical point acquisition, and integration of respiratory compensation algorithms. ${ }^{20}$ Potential solutions include temporarily decreasing the speed of the motor and/or obtaining location-only points using the impedance mapping function of the mapping system. ${ }^{19-21}$ Predisposing factors for EMI, quantification of EMI in relation to these factors and potential solutions have not been systematically evaluated. The EnSite Velocity system using its EnSite NavX application (St. Jude Medical Inc., St. Paul, MN, USA) is an electrical impedance-based mapping system, which may result in lower incidence of EMI.

We sought to characterize the incidence, severity, predisposing factors, and potential solutions for EMI while using a microaxial flow device with a magnet-based mapping system and an electrical impedance-based system in a canine model during sinus rhythm, ventricular pacing, and ventricular fibrillation (VF). 


\section{Methods}

\section{Experimental preparation}

Seven healthy male hounds with mean weight of $32.4+/-2.44 \mathrm{~kg}$ were used for the study. The study protocol was approved by the Mayo Foundation Institutional Animal Care and Use Committee. Study subjects were premedicated with ketamine $(10 \mathrm{mg} / \mathrm{kg})$ and diazepam $(0.5 \mathrm{mg} / \mathrm{kg})$. Isoflurane was used for maintenance of anesthesia at $1 \%$ to $5 \%$ inhalation. Electrocardiograms, local electrograms, and arterial blood pressure were continuously monitored using the CardioLab System (GE Healthcare, Wauwatosa, WI, USA).

Percutaneous endovascular access was obtained via the right external jugular vein (12 Fr), right femoral vein $(8 \mathrm{Fr})$, right femoral artery ( $8 \mathrm{Fr}$ ), and left femoral artery (13 Fr). Transseptal catheterization was performed under ultrasound and fluoroscopic guidance through the 8-Fr femoral venous sheath. Pericardial access was obtained through the subxiphoid approach under fluoroscopic guidance using the techniques described by Scanavacca et al. ${ }^{22}$ Intracardiac echocardiography (Siemens TM, Mountain View, CA, USA) was performed through a catheter placed in the right atrium from the right external jugular vein. The microaxial flow device was placed in the left ventricle through the retrograde aortic approach as described previously. ${ }^{22}$ The MFD was operated at the maximum performance level (P8, flow rate 1.9-2.5 L/min). The MFD can be operated at various performance levels, with each performance level corresponding to certain revolutions per minute of the impeller (Supp Table 1).

Mapping of the endocardial and epicardial aspects of the right ventricle (RV) and left ventricle (LV) was performed through the right femoral vein, retrograde aortic, and pericardial approach using an 8-Fr bi-directional ThermoCool irrigated ablation catheter (Biosense Webster, CA). Mapping was performed sequentially during sinus rhythm and right ventricular pacing in all animals and induced VF in 2 animals. Electroanatomical maps of all the chambers were created twice using the impedance-based and magnet-based mapping systems.

The presence of EMI with mapping, reproducibility of the map, and the integrity of recorded electrical signals was noted. When EMI occurred, interference was recorded as severe EMI when alerts were displayed and mapping catheter could not be reliably localized; and as mild EMI when alerts were displayed but mapping catheter could be reliably localized. Statistical comparisons were made between the severe EMI group and the mild/no interference group. The MFD itself was mapped in 2 subjects. A representative point along the left ventricular outflow tract, which corresponded to the location of the impeller, was chosen as a reference point. The distance between the mapping catheter and this reference point was calculated for all points acquired. When interference with mapping was observed, two troubleshooting procedures as described below were followed.

\section{EMI Troubleshooting}

Two methods of troubleshooting were tested: 1) titration of MFD performance, and 2) the use of impedance only mapping in conjunction with manual annotation of local activation times. The presence of interference, reproducibility of the map, and integrity of the electrical 
signals was once again noted following both procedures. The distance of the mapped points was measured if the interference resolved.

The procedure for obtaining impedance-only points with the Carto3 system is demonstrated in the supplemental material. Briefly, this method allows for visualization of the catheter location but not local electrograms or activation times. Once the catheter location is obtained, the CardioLab system can be used to obtain the local activation time. This time is then manually annotated to the location on the map.

\section{Statistical analysis}

Statistical analysis was performed using JMP 9.0.1 software (SAS, Cary, NC, USA). Continuous variables are represented as mean \pm standard deviation, and compared using Students t-test. Categorical variables are represented as percentages, and compared with Chi-square test. Two-tailed P-values of $₫ \mathbf{0 . 0 5}$ were considered statistically significant.

\section{Results}

All 7 dogs were mapped during sinus rhythm and ventricular pacing, and 2 dogs were mapped in VF. The degree of interference for each point acquired was recorded for 5 dogs. The mean duration of VF was 68.6 ( \pm 52.9$)$ minutes. No premature deaths occurred, and the microaxial flow device was successful in maintaining cardiac output through the study.

\section{Mapping interference with electrical impedance based mapping system}

Detailed endocardial and epicardial mapping was possible during sinus rhythm, ventricular pacing, and also during VF using the electrical impedance-based mapping system without EMI, with the microaxial flow device at P8 performance level in all 7 dogs. In total, 308 points were acquired (71 RV points, $75 \mathrm{LV}$ points and 162 epicardial points) without any EMI. There was no mechanical interference of microaxial flow device with the endocardial mapping catheters.

\section{Mapping interference with magnet-based mapping}

Endocardial and epicardial mapping during sinus rhythm, RV pacing, and during VF was subject to EMI while using the magnet-based mapping system, with the microaxial flow device at $\mathrm{P} 8$ performance level (Figure 1). Out of a total 673 points acquired in $5 \mathrm{dogs}$, severe EMI was observed in $9.4 \%$ of all points, mild EMI in 58.5\% points and no interference in $32.1 \%$ points. Severe EMI occurred with endocardial mapping (11.1\%), epicardial mapping (6.9\%), LV mapping (13.3\%), and RV mapping (9.1\%). There was a nonsignificant trend toward severe interference in the endocardial mapping group compared to the epicardial mapping group $(11.1 \%$ vs $6.9 \%, \mathrm{p}=0.062)$. There was no difference in severe EMI between the RV and LV mapping groups (Table 1).

The mean distance of points from the MFD with severe EMI was $40.1 \mathrm{~mm}( \pm 16.8 \mathrm{~mm})$, with mild EMI was $52.7 \mathrm{~mm}( \pm 18.5 \mathrm{~mm})$, and with no EMI was $60.5 \mathrm{~mm}( \pm 21.4 \mathrm{~mm})$. Severe EMI occurred at points that were significantly closer to the MFD compared to points with mild/no EMI that were further away, and this was observed irrespective of the mapping 
chamber (LV or RV; endocardial or epicardial). Endocardial points were significantly closer to the MFD than epicardial points with a mean difference in distance of $12.6 \mathrm{~mm}$ (CI 9.6$15.6 \mathrm{~mm}, \mathrm{p}<0.0001$ ) (Table 2).

\section{Troubleshooting EMI with the magnet-based mapping system}

Adjustment of MFD speed-All points with severe interference resolved to either mild interference or no interference with change in MFD performance from P8 to P6. This resolution was not consistently observed with change in performance from P8 to P7, P5, or even lower performance levels.

\section{Impedance-based location mapping and manual annotation of activation time}

-Impedance-only mapping coupled with manual annotation of activation times on the map was also successful for all points with severe EMI. MFD performance level was maintained at P8 during impedance-only mapping.

\section{Discussion}

We report for the first time the frequent incidence of EMI while using the MFD in conjunction with the magnet-based electroanatomical mapping system. While EMI is mild in most cases, severe EMI occurs frequently and leads to difficulties in accurate catheter localization and electroanatomical point acquisition. ${ }^{20}$ Proximity to the microaxial flow device and maximal MFD performance are identified as predisposing factors for EMI with the use of magnet-based mapping system. Electrical impedance-based mapping with the EnSite NavX system results in very infrequent and mild EMI, which does not preclude detailed endocardial and epicardial mapping.

Decreasing the performance level of the MFD from P8 $(1.9-2.5 \mathrm{~L} / \mathrm{min})$ to P6 $(1.4-2.0 \mathrm{~L} /$ min) resulted in resolution of severe EMI in all cases. The advanced mapping features of the predominantly magnet-based Carto3 mapping system permit electrical impedance-based mapping, which can be used to map very close to the MFD. This method allows for point acquisition in all cases of severe interference. This method is described in depth in the supplementary materials.

\section{EMI associated with the use of microaxial flow device}

Previous studies have noted that EMI occurs with the use of microaxial flow device and magnet-based electroanatomical mapping systems. ${ }^{19-21}$ EMI was observed in 9 out of 20 patients in one study and 10 out of 22 patients in another study. The degree of EMI experienced was mild in most cases and was not prohibitive for detailed electroanatomical mapping. Reduction of MFD performance level from P8 (1.9-2.5 L/min) to P2 (0.4-1.0 L/ $\mathrm{min}$ ) resulted in resolution of interference in most cases in these studies. The authors note that interference occurred despite performance reduction at points very close to the MFD. They also note that impedance-only mapping can be utilized to map such points. However, these studies were not designed to systematically evaluate the incidence of EMI, the predisposing factors, and the solutions for EMI. 
In our study, EMI occurred frequently with the magnet-based system, with $9.4 \%$ of all points resulting in severe EMI. There was a significant number of instances with severe EMI that prohibited accurate electroanatomic mapping. Electrical impedance-based systems had very infrequent instances of EMI, and accurate endocardial and epicardial maps could be obtained without having to titrate MFD performance.

\section{Predisposing factors for EMI with magnet based mapping}

Miller et al. have noted that EMI is dependent on distance between the microaxial flow device motor and the mapping catheter. ${ }^{20,21}$ EMI was observed when mapping in the ventricular outflow tracts close to the microaxial flow device motor. Our study establishes that distance between the MFD motor and the tip of the mapping catheter is a significant predictor of EMI. Comparison between distance from MFD for points with severe and mild/no EMI demonstrates the effect of distance on the severity of the EMI. Points with severe interference were consistently farther than points with no or mild interference, irrespective of anatomic location. A trend toward higher incidence of severe EMI was observed during endocardial mapping in comparison to epicardial mapping. This result can be expected due to greater proximity to the MFD's impeller during endocardial mapping.

Miller et al. have noted a "dose-dependent" relationship between MFD pump performance and EMI, with performance reduction from P8 to P2, resulting in resolution of most interference. ${ }^{20}$ Our findings support that pump performance is a significant predictor of EMI. All occurrences of severe EMI occurred at P8 performance level (flow rate 1.9- 2.5 L/ $\mathrm{min})$. Reduction of performance level to P6 (1.4-2.0 L/min) resulted in resolution of EMI in all cases. The reduction of performance was hemodynamically well-tolerated. We noted, however, that EMI did not resolve consistently with MFD settings other than P6. This suggests a close correlation between the occurrence of EMI and the frequency of rotation of the MFD. We hypothesize that the electrical frequency applied for rotation of the impeller motor at the P6 performance level (45,000 RPM) is filtered out by the magnet-based mapping system.

\section{Solutions for EMI with magnet-based mapping system}

Electrical impedance-based mapping systems do not result in EMI with MFD. It may be difficult for established practices to change the operating system. Our study emphasizes two potential solutions to resolve EMI with magnet-based systems. These have been recognized previously, but have not been described in depth. ${ }^{19}$

Temporarily decreasing microaxial flow device performance level from P8 to P6 allowed the magnet-based mapping system to obtain point locations and electrical activity for all 70 points for which severe EMI was observed. Measures of perfusion such as mean arterial pressure and atrial contractility were well-maintained at the P6 performance level.

This study also outlined and evaluated a procedure to perform high-resolution electroanatomic mapping that overcomes EMI, independent of MFD performance level. This method requires combining location-only points obtained using the impedance mapping feature available through the Carto3 system and local electrical activation times obtained through the CardioLab system. Location-only points were obtained for all points 
with severe interference, and electroanatomical mapping was completed successfully in all subjects. The loss of image resolution with this method was minimal and resulted in accurate electroanatomical maps with maximal MFD performance.

\section{Limitations}

This is a small animal study to identify determinants and solutions for EMI with magnetbased and impedance-based mapping. The results may not be generalizable to human subjects, especially with presence of myocardial scarring. In humans, change in performance level of the MFD may not completely eliminate noise or may do so at different performance levels. We hope that the findings of our study will provide the basis for a future trial to confirm these data in humans.

Regardless, our study found similar solutions to those proposed in earlier human studies, including reduction in the performance level of MFD to P6 or using impedance-based location mapping. However, concerns for maintaining adequate cardiac support exist even at performance level P8 in humans, ${ }^{20}$ and titration of MFD performance in real life scenarios may not always be possible.

\section{Conclusion}

EMI occurs frequently with the concurrent use of a microaxial flow device and a magnetbased mapping system. Severe interference prohibiting accurate mapping occurs more frequently at maximal performance level, and is a function of distance between the MFD motor and the mapping catheter tip. Potential solutions to overcome EMI during use of a magnet-based mapping system include decreasing MFD performance and utilizing a combination of impedance-only mapping and manual annotation of local electrical activation. Further investigation is required to confirm the applicability of our findings to human cases with unstable ventricular tachycardia. Electric impedance-based mapping systems do not result in EMI, and generate accurate electroanatomical maps at maximum MFD performance.

\section{Supplementary Material}

Refer to Web version on PubMed Central for supplementary material.

\section{Acknowledgments}

St. Jude Medical has provided moderate $(\$ 10,000-100,000)$ financial support for this research.

\section{Abbreviations}

$\begin{array}{ll}\text { EMI } & \text { Electromagnetic interference } \\ \text { MFD } & \text { Microaxial flow device } \\ \text { LV } & \text { Left ventricle } \\ \text { RV } & \text { Right ventricle }\end{array}$




\section{References}

1. Bansch D, Oyang F, Antz M, Arentz T, Weber R, Val-Mejias JE, Ernst S, Kuck KH. Successful catheter ablation of electrical storm after myocardial infarction. Circulation. 2003; 108:3011-3016. [PubMed: 14662718]

2. Moss AJ, Greenberg H, Case RB, Zareba W, Hall WJ, Brown MW, Daubert JP, McNitt S, Andrews ML, Elkin AD. Long-term clinical course of patients after termination of ventricular tachyarrhythmia by an implanted defibrillator. Circulation. 2004; 110:3760-3765. [PubMed: 15583079]

3. Reddy VY, Reynolds MR, Neuzil P, Richardson AW, Taborsky M, Jongnarangsin K, Kralovec S, Sediva L, Ruskin JN, Josephson ME. Prophylactic catheter ablation for the prevention of defibrillator therapy. N Engl J Med. 2007; 357:2657-2665. [PubMed: 18160685]

4. Stevenson WG, Soejima K. Catheter ablation for ventricular tachycardia. Circulation. 2007; 115:2750-2760. [PubMed: 17533195]

5. Yamada T, Kay GN. Optimal ablation strategies for different types of ventricular tachycardias. Nat Rev Cardiol. 2012; 9:512-525. [PubMed: 22641339]

6. Zipes DP, Camm AJ, Borggrefe M, Buxton AE, Chaitman B, Fromer M, Gregoratos G, Klein G, Moss AJ, Myerburg RJ, Priori SG, Quinones MA, Roden DM, Silka MJ, Tracy C, Smith SC Jr, Jacobs AK, Adams CD, Antman EM, Anderson JL, Hunt SA, Halperin JL, Nishimura R, Ornato JP, Page RL, Riegel B, Blanc JJ, Budaj A, Dean V, Deckers JW, Despres C, Dickstein K, Lekakis J, McGregor K, Metra M, Morais J, Osterspey A, Tamargo JL, Zamorano JL. ACC/AHA/ESC 2006 Guidelines for Management of Patients With Ventricular Arrhythmias and the Prevention of Sudden Cardiac Death: a report of the American College of Cardiology/American Heart Association Task Force and the European Society of Cardiology Committee for Practice Guidelines (writing committee to develop Guidelines for Management of Patients With Ventricular Arrhythmias and the Prevention of Sudden Cardiac Death): developed in collaboration with the European Heart Rhythm Association and the Heart Rhythm Society. Circulation. 2006; 114:e385-e484. [PubMed: 16935995]

7. Marchlinski FE, Callans DJ, Gottlieb CD, Zado E. Linear ablation lesions for control of unmappable ventricular tachycardia in patients with ischemic and nonischemic cardiomyopathy. Circulation. 2000; 101:1288-1296. [PubMed: 10725289]

8. Arenal A, Glez-Torrecilla E, Ortiz M, Villacastin J, Fdez-Portales J, Sousa E, del Castillo S, Perez de Isla L, Jimenez J, Almendral J. Ablation of electrograms with an isolated, delayed component as treatment of unmappable monomorphic ventricular tachycardias in patients with structural heart disease. J Am Coll Cardiol. 2003; 41:81-92. [PubMed: 12570949]

9. Connolly SJ, Dorian P, Roberts RS, Gent M, Bailin S, Fain ES, Thorpe K, Champagne J, Talajic M, Coutu B, Gronefeld GC, Hohnloser SH. Comparison of beta-blockers, amiodarone plus betablockers, or sotalol for prevention of shocks from implantable cardioverter defibrillators: the OPTIC Study: a randomized trial. JAMA. 2006; 295:165-171. [PubMed: 16403928]

10. Friedman PA, Asirvatham SJ, Grice S, Glikson M, Munger TM, Rea RF, Shen WK, Jahanghir A, Packer DL, Hammill SC. Noncontact mapping to guide ablation of right ventricular outflow tract tachycardia. J Am Coll Cardiol. 2002; 39:1808-1812. [PubMed: 12039496]

11. Ganz LI, Stevenson WG. Catheter mapping and ablation of ventricular tachycardia. Coron Artery Dis. 1996; 7:29-35. [PubMed: 8773430]

12. Hsia HH, Callans DJ, Marchlinski FE. Characterization of endocardial electrophysiological substrate in patients with nonischemic cardiomyopathy and monomorphic ventricular tachycardia. Circulation. 2003; 108:704-710. [PubMed: 12885746]

13. Marchlinski FE, Zado E, Dixit S, Gerstenfeld E, Callans DJ, Hsia H, Lin D, Nayak H, Russo A, Pulliam W. Electroanatomic substrate and outcome of catheter ablative therapy for ventricular 
tachycardia in setting of right ventricular cardiomyopathy. Circulation. 2004; 110:2293-2298. [PubMed: 15477406]

14. Abuissa H, Roshan J, Lim B, Asirvatham SJ. Use of the Impella microaxial blood pump for ablation of hemodynamically unstable ventricular tachycardia. J Cardiovasc Electrophysiol. 2010; 21:458-461. [PubMed: 20039989]

15. Bunch TJ, Darby A, May HT, Ragosta M, Lim DS, Taylor AM, DiMarco JP, Ailawadi G, Revenaugh JR, Weiss JP, Mahapatra S. Efficacy and safety of ventricular tachycardia ablation with mechanical circulatory support compared with substrate-based ablation techniques. Europace. 2012; 14:709-714. [PubMed: 22080473]

16. Bunch TJ, Mahapatra S, Madhu Reddy Y, Lakkireddy D. The role of percutaneous left ventricular assist devices during ventricular tachycardia ablation. Europace. 2012; 14(Suppl 2):ii26-ii32. [PubMed: 22832915]

17. Carbucicchio C, Della Bella P, Fassini G, Trevisi N, Riva S, Giraldi F, Baratto F, Marenzi G, Sisillo E, Bartorelli A, Alamanni F. Percutaneous cardiopulmonary support for catheter ablation of unstable ventricular arrhythmias in high-risk patients. Herz. 2009; 34:545-552. [PubMed: 20091254]

18. Friedman PA, Munger TM, Torres N, Rihal C. Percutaneous endocardial and epicardial ablation of hypotensive ventricular tachycardia with percutaneous left ventricular assist in the electrophysiology laboratory. J Cardiovasc Electrophysiol. 2007; 18:106-109. [PubMed: 17229307]

19. Miller MA, Dukkipati SR, Chinitz JS, Koruth JS, Mittnacht AJ, Napolitano C, d'Avila A, Reddy VY. Percutaneous hemodynamic support with Impella 2.5 during scar-related ventricular tachycardia ablation (PERMIT 1). Circ Arrhythm Electrophysiol. 2013; 6:151-159. [PubMed: 23255277]

20. Miller MA, Dukkipati SR, Koruth JS, d'Avila A, Reddy VY. How to perform ventricular tachycardia ablation with a percutaneous left ventricular assist device. Heart Rhythm. 2012; 9:1168-1176. [PubMed: 22322326]

21. Miller MA, Dukkipati SR, Mittnacht AJ, Chinitz JS, Belliveau L, Koruth JS, Gomes JA, d'Avila A, Reddy VY. Activation and entrainment mapping of hemodynamically unstable ventricular tachycardia using a percutaneous left ventricular assist device. J Am Coll Cardiol. 2011; 58:13631371. [PubMed: 21920266]

22. Sosa E, Scanavacca M, d'Avila A, Pilleggi F. A new technique to perform epicardial mapping in the electrophysiology laboratory. J Cardiovasc Electrophysiol. 1996; 7:531-536. [PubMed: 8743758] 


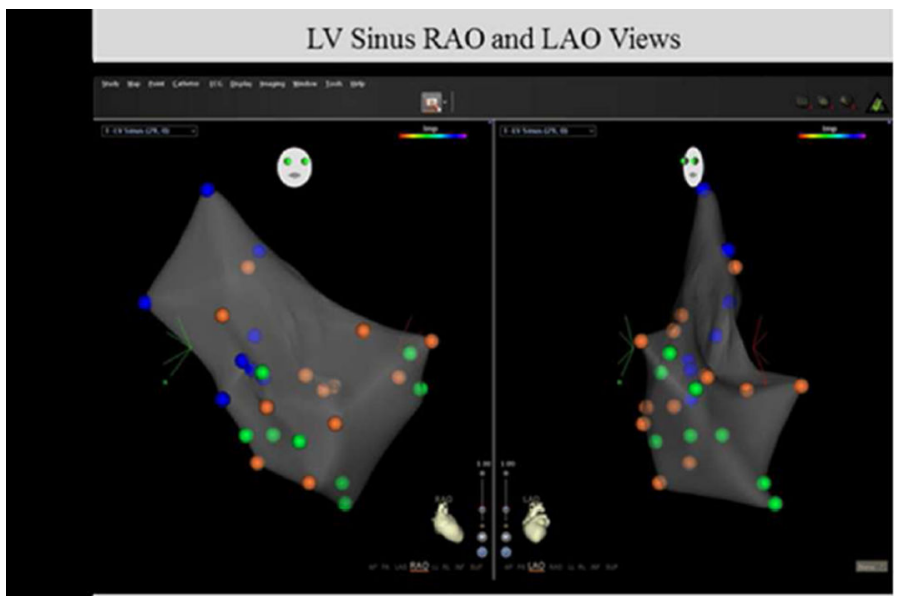

$\underline{\text { RV Sinus RAO and LAO Views }}$

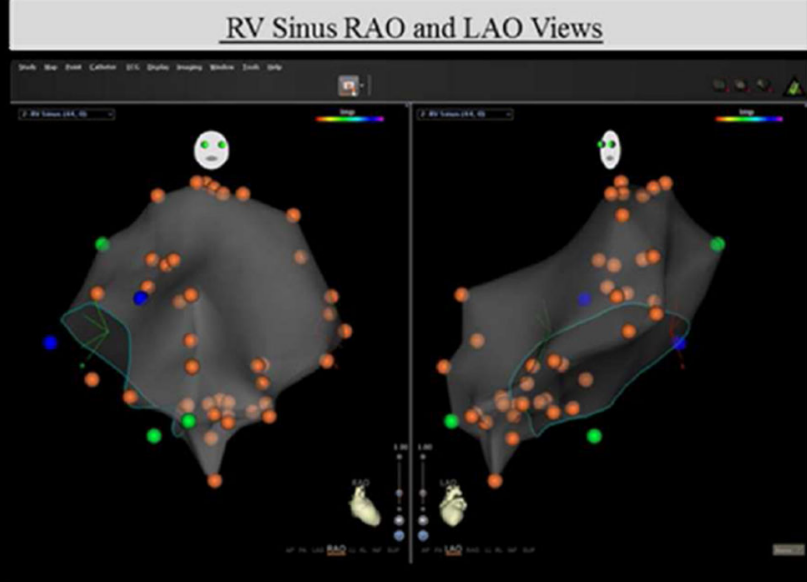

Epicardial Sinus RAO and LAO Views
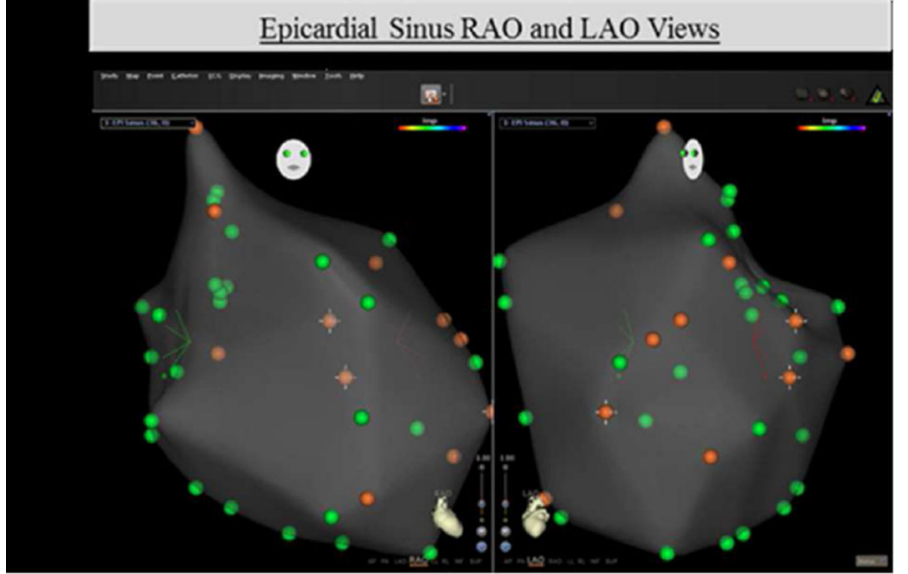

Figure 1.

Right (a) and left (b) ventricular endocardial mapping, epicardial mapping (c) in sinus rhythm with the MFD at maximal performance using the magnet-based mapping system. Blue points: severe distortion, orange points: mild distortion, green points: no distortion. Endocardial maps $(a, b)$ have greater numbers of blue dots compared with the epicardial map (c); indicating greater occurrence of severe distortion with endocardial maps. 


\section{Table 1}

Distribution of points acquired using magnet-based mapping by electromagnetic interference caused with concurrent use of microaxial flow left ventricular assist device at P8 performance level.

\begin{tabular}{lllll}
\hline & Severe EMI & p-value $^{*}$ & Mild EMI & No EMI \\
\hline All points (n=673) & $\mathbf{6 3}(\mathbf{9 . 4 \%})$ & & $394(58.5 \%)$ & $216(32.1 \%)$ \\
\hline Endocardial points $(\mathbf{n = 3 9 7 )}$ & $\mathbf{4 4}(\mathbf{1 1 . 1 \%})$ & & $282(71.0 \%)$ & $71(17.9 \%)$ \\
Epicardial points (n=276) & $\mathbf{1 9}(\mathbf{6 . 9 \% )}$ & & $112(40.6 \%)$ & $145(52.5 \%)$ \\
\hline LV points (n=188) & $\mathbf{2 5 ( 1 3 . 3 \% )}$ & $120(63.8 \%)$ & $43(22.9 \%)$ \\
RV points $(\mathbf{n = 2 0 9})$ & $\mathbf{1 9}(\mathbf{9 . 1 \%})$ & & $162(77.5 \%)$ & $28(13.4 \%)$ \\
\hline
\end{tabular}

* Comparing severe interference between groups

Severe EMI, interference in acquiring points; mild EMI, system interference alerts without interference in acquiring points; EMI, electromagnetic interference; LV, left ventricle; RV, right ventricle 


\section{Table 2}

Comparison of mean distance from microaxial left ventricular assist device at P8 performance level to locations having severe compared to mild/no interference with magnet-based mapping.

\begin{tabular}{llll}
\hline & Severe EMI & Mild/no EMI & p-value \\
\hline All points (n=673) & $40.1 \pm 16.8$ & $55.5 \pm 20.0$ & $<0.0001$ \\
\hline Endocardial points $(\mathbf{n = 3 9 7 )}$ & $34.7 \pm 14.3$ & $50.6 \pm 18.8$ & $<0.0001$ \\
Epicardial points $(\mathbf{n = 2 2 4 )}$ & $52.4 \pm 15.9$ & $62.1 \pm 19.5$ & $<0.0001$ \\
\hline LV points $(\mathbf{n = 1 8 8})$ & $35.5 \pm 17.1$ & $51.6 \pm 19.1$ & $<0.0001$ \\
RV points $(\mathbf{n = 2 0 9})$ & $33.7 \pm 9.9$ & $49.8 \pm 18.7$ & $<0.0001$ \\
\hline
\end{tabular}

